\section{Kompass \\ Ophthalmologie}

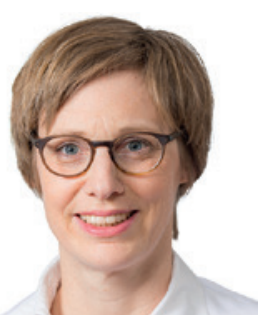

\section{Ameli Gabel-Pfisterer}

Klinik für Augenheilkunde, Ernst von Bergmann Klinikum, Potsdam, Deutschland

\title{
Augenverletzungen durch Pyrotechnik
}

Was als erwartungsfrohes Fest mit Freunden und Familie beginnt, endet immer wieder dramatisch: Verletzungen durch privates Feuerwerk betreffen jedes Jahr nicht nur die, die Feuerwerk selbst zünden, sondern auch unbeteiligte Zuschauer oder Passanten.

Da bislang unklar war, wie häufig solche Verletzungen sind, und gleichzeitig eine Diskussion um ein Verbot privater Feuerwerke hörbar wurde, haben wir im Jahr 2016 als gemeinsame Arbeitsgruppe aus Potsdam und Freiburg im Breisgau unsere deutschlandweite Studie zur Häufigkeit und Schwere, zu Unfallhergang und Therapiebedarf nach Augenverletzungen durch Pyrotechnik initiiert. Mit Hilfe der diensthabenden AugenärztInnen, die neben der Versorgung der Verletzten die Daten in den Online-Fragebogen eingegeben haben, können wir die Situation inzwischen genauer beschreiben. Vielen Dank allen Beteiligten für Ihr Engagement, machen Sie bitte auch in Zukunft mit!
Die Dreijahres-Daten haben wir 2019 erstmals veröffentlicht, sie sind in dieser Ausgabe erneut einzusehen. Im vierten Jahr unserer Umfrage an Silvester 2019/2020 gab es in den Daten wenig Veränderung im Vergleich zu den Vorjahren: Erneut waren mehr als die Hälfte der rund 500 Verletzten Zuschauer oder Passanten, Kinder und Jugendliche deutlich überrepräsentiert, und knapp ein Viertel aller Patienten musste stationär behandelt werden. Wir sahen daher in den Daten eine Konsistenz über Jahre - bis die Corona-Pandemie am Jahreswechsel 2020/2021 ein Verkaufsverbot für private Feuerwerkskörper und weitreichende Versammlungsbeschränkungen notwendig machte und die Anzahl der registrierten Patienten auf 79 zurückging. Dies hat uns zum einen die Sinnhaftigkeit unserer longitudinalen Beobachtung und zum anderen den protektiven Effekt einer Reduktion der privaten Feuerwerke gezeigt.

Einen Überblick über Feuerwerksverletzungen anderer Körperregionen gibt der Artikel von Van Yperen. Hier werden 10-Jahresdaten aus zwei niederländischen Traumazentren im Hinblick auf Verletzungsmuster und auslösende Pyrotechnik zusammengefasst. Es wird deutlich, dass Knallkörper eher zu Verletzungen der oberen Extremitäten führen, während aufsteigende Feuerwerkskörper, wie Raketen, überwiegend Verletzungen an Kopf und Hals verursachen. Beide Gruppen von Pyrotechnik sind für die hier dokumentierten Augenverletzungen bei 20 Patienten, mit einer Erblindung von 6 Augen, verantwortlich.

Die Lektüre beider Artikel zeigt, wie folgenreich Feuerwerksverletzungen der Hände, des Gesichts und der Augen sein können. Lassen Sie sich von den Daten überzeugen, und unterstützen Sie uns bei der Forderung nach sicherem Feuerwerk!

Lesen Sie dazu auch auf der Homepage der DOG (www.dog.org) unsere Vorschläge zu kommunalem Feuerwerk.

Herzlich,

Ihre A. Gabel-Pfisterer 